\title{
Polarity of Constitutive and Regulated von Willebrand Factor Secretion by Transfected MDCK-II Cells
}

\author{
Caroline Hop, Ruud Fontijn, J an A. van Mourik,* and Hans Pannekoek ${ }^{1}$ \\ Department of Biochemistry, University of Amsterdam, Academic Medical Center, 1105 AZ Amsterdam, The Netherlands; and \\ *Department of Blood Coagulation, Central Laboratory of the Netherlands Red Cross Blood Transfusion Service, \\ 1066 CX Amsterdam, The Netherlands
}

\begin{abstract}
Von Willebrand factor (vWF), synthesized by endothelial cells, is both rapidly secreted by the constitutive pathway and stored in Weibel - Palade bodies. Secretion from these organelles occurs upon activation of the protein kinase $\mathrm{C}$ signal transduction pathway and yields highly multimerized vWF. Highly multimerized vWF acts as a more effective adhesive ligand than the lower molecular weight forms that are constitutively secreted. We employed the extensively characterized polar Madin-Darby Canine Kidney II (MDCKII) epithelial cell line, stably transfected with fulllength vWF CDNA or deletion mutants thereof, to gain insight in the polarity of vWF secretion by either one of the two pathways. Immunofluorescence analysis and metabolic labeling experiments revealed that multimeric "wild-type" vWF is stored in MDCK-II cells and released upon stimulation with phorbol esters. Furthermore, we show that $\mathbf{6 2 . 0} \pm \mathbf{3 . 8 \%}$ of constitutively secreted and $83.2 \pm 6.6 \%$ of the regulated secreted wildtype vWF is encountered at the apical side of the cell. The polarity of the constitutive secretion of deletion mutant vWF delD'D3 is similar to that of constitutively secreted wild-type vWF, whereas deletion mutant vWF delD1D2 displays no polar secretion (50.1 $\pm \mathbf{5 . 7 \%}$ apical). 1997 Academic Press
\end{abstract}

\section{INTRODUCTION}

Von Willebrand factor $(V W F)^{2}$ is a multimeric glycoprotein that is essential both for the adhesion of platel ets to exposed subendothelium and for the sub-

\footnotetext{
${ }^{1}$ To whom correspondence and reprint requests should be addressed. Fax: 31-(0)20-6915519.

${ }^{2}$ Abbreviations used: BSA, bovine serum al bumin; CLSM, confocal scanning laser microscopy; ELISA, enzyme-linked immunosorbent assay; FITC, fluorescein isothiocyanate; MDCK-II, Madin Darby caninekidney-II cells; VWF, von Willebrand factor; PBS, $10 \mathrm{mM}$ sodium phosphate ( $\mathrm{pH} 7.4), 0.14 \mathrm{M} \mathrm{NaCl}$; PDB, phorbol dibutyrate; PMA, phorbol myristate acetate; RER, rough endoplasmatic reticulum, SDS-PAGE, sodium dodecylsulfate- polyacrylamide gel el ectrophoresis; u-PA, urokinase-type plasminogen activator.
}

sequent aggregation of platelets [1, 2]. The expression of vWF is restricted to two cell types, i.e., the megakaryocyte and the endothelial cell. The biosynthesis and secretion of VWF has been extensively studied in cultured endothel ial cells [3]. I n summary, VWF is synthesized as a precursor protein (pre-proVWF ) that consists of a signal peptide, followed by a pro-polypeptide and mature vWF of 741 and 2050 amino-acid residues, respectively $[4,5]$. The precursor protein is a highly repetitive molecule, composed of four different domains (A, B, C, and D) that are arranged in the foll owing order: D1-D2-D'-D3-A1-A2A3-D4-B1-B2-B3-C1-C2. Pro-vWF monomers are assembled into pro-vWF dimers in the rough endoplasmatic reticulum (RER) [6-9] and after transport to the Golgi apparatus, a series of multimers is formed [3]. Multimerization requires the presence of the domains D1 and D2 (constituting the pro-polypeptide) and the amino-terminal $\mathrm{D}^{\prime}$ and D3 domains of mature vWF [10].

In cultured cells, approximately $5 \%$ of de novo-synthesized VWF is sorted into endothelial cell-specific storage organelles, the so-called Weibel-Palade bodies [11], whereas the remaining $95 \%$ of the protein is packaged into secretory vesicles and rapidly secreted by the constitutive pathway [12]. Release of VWF, stored in the Weibel-Palade bodies, can be induced by various agents, including thrombin, cytokines, or phorbol esters [6, 13, 14]. This secretory route is denoted the regulated pathway [15]. Constitutively secreted VWF consists of dimers and low molecular weight multimers that are partially proteolytically processed. In contrast, vWF originating from theWeibel - Palade bodies predominantly consists of high molecular weight multimers that are completely proteolytically processed $[12,14,16]$. The choice for a particular secretory pathway dictates the adhesive quality of vWF. This is exemplified by the observation that the affinity of high molecular weight multimers for glycoprotein Ib, the VWF receptor on platelets, is about 100 -fold higher than that of low molecular weight species [17].

To study the requirements for polar secretion of VWF, ideally a cell-type should be chosen that: (i) has been thor- 
oughly characterized with regard to its polar growth properties, (ii) does not synthesize endogenous WWF and, (iii) can be stably transfected with "wild-type" vWF cDNA and variants thereof. The latter feature would allow to assess the contribution of different domains of VWF in routing of the protein. These criteria are met in the present study, using the extensively, characterized Madin-Darby Canine Kidney-II (MDCK-II) cell line [18-20]. These cells have been employed to analyze the polar secretion of a number of secretory proteins, such as urokinase-type plasminogen activator (U-PA) which is predominantly secreted to the apical compartment $[21,22]$.

Our previous studies indicate that storage of VWF in Weibel-Palade-like organelles is governed by intracellular multimerization and is not restricted to a particular (transfected) cell type. Consequently, we used different VWF cDNA constructs either to allow multimerization (full-length VWF CDNA) or to prevent the formation of VWF multimers by the deletion of specific domains (i.e., D1 and D2 or D' and D3) [9, 10, 23]. As a result a model system was created to investigate the pathways and polarity of vWF secretion.

\section{MATERIALS AND METHODS}

Tissue culture and transfection. MDCK-II cells (kindly provided by Dr. G. van Meer, Academic Medical Hospital, Utrecht, The Netherlands) were maintained in I scove's modified minimal medium, supplemented with $100 \mathrm{U} / \mathrm{ml}$ penicillin, $100 \mu \mathrm{g} / \mathrm{ml}$ streptomycin, $20 \mu \mathrm{l} /$ $\mathrm{ml}$ fungizone, and $10 \%(\mathrm{v} / \mathrm{v}$ ) fetal calf serum (Gibco Laboratories, Paisly, UK). Transfections were performed using the calcium phosphate precipitation method [2] to integrate either pcRSVvWF, pcRSV VWF delD1D2, or pcRSV vWF delD'D3 into the genome of the cells. These constructs have been described previously [9, 10]. The vector part of these plasmids carries neomycin resistance allowing a selection for 10 days on culturing medium that contains $400 \mu \mathrm{g} /$ $\mathrm{ml}$ of neomycin (G418).

Immunofluorescence. Cells were grown to confluency on glasscoverslips coated with fibronectin or on polycarbonate filters. Then, the cells were washed twice with $10 \mathrm{mM}$ sodium phosphate $(\mathrm{pH}$ 7.4), $0.14 \mathrm{M} \mathrm{NaCl}$ (PBS), and subsequently fixed for $10 \mathrm{~min}$ at room temperature with methanol. After fixation, the cells were washed twice with $\mathrm{PBS}$ and incubated for $1 \mathrm{~h}$ with a mixture of the murine anti-human vWF monoclonal antibodies CLB-RAg 201, CLB-RAg 35, and CLB-RAg 21 (Central Laboratory of the Netherlands Red Cross Blood Transfusion Service (Amsterdam, The Netherlands)) and/or with a rabbit anti-human cathepsin D polyclonal antiserum in PBS, supplemented with $3 \%(\mathrm{w} / \mathrm{v})$ bovine serum albumin (BSA; Organon Technika, Boxtel, The Netherlands) and $0.1 \%(v / v)$ Tween 20 . Finally, the coverslips were washed twice with PBS and incubated for $1 \mathrm{~h}$ with a cy-3-labeled conjugate of goat anti-rabbit immunoglobulins and/or a fluorescein isothiocyanate (FITC)-labeled conjugate of goat anti-mouse immunoglobulins in PBS supplemented with $3 \%(\mathrm{w} / \mathrm{v})$ BSA. After extensive washing with PBS, the coverslips were prepared for microscopy by embedding in mounting medium (vecta shield, Sigma, St. Louis, MO) and were analyzed by fluorescence microscopy or by confocal scanning laser microscopy (CLSM) (Leica, Heidelberg, Germany).

VWF multimer analysis. VWF-containing conditioned media of MDCK-II cell lines, that were cultured at confluency for $24 \mathrm{~h}$, were centrifuged for $5 \mathrm{~min}$ at $500 \mathrm{~g}$ and subsequently concentrated by fil- tration on Centricon-30 filters (Amicon, Danvers, MA). The analysis of VWF multimers was performed as described [25].

Metaboliclabeling. Stably transfected MDCK-II cells were grown to confluency in $80-\mathrm{cm}^{2}$ culture flasks, harvested by trypsinization, seeded (surface dilution 1:0.75) on 4.6- $\mathrm{cm}^{2}$ polycarbonate filters (pore size, $0.4 \mu \mathrm{m}$ ) of a Transwell (Costar, Badhoevedorp, The Netherlands), and grown for 4 days in Iscove's modified minimal medium [26]. After washing with PBS, the cells were labeled for 3 days by adding the following components to the basolateral compartment: 85 $\mu \mathrm{Ci}{ }^{35} \mathrm{~S}$-labeled amino acids (containing 65\% methionine and $25 \%$ cysteine; Radiochemical Centre, Amersham, UK) in Dulbecco's modified Eagle medium, supplemented with L-arginine (84 mg/liter), Lglutamine (584 mg/liter), L-leucine (105 mg/liter), glucose (4.5 $\mu \mathrm{g} /$ liter), inositol (7.2 mg/liter), sodium phosphate (125 mg/liter), and $10 \%(\mathrm{v} / \mathrm{v})$ of dialyzed fetal calf serum. Subsequently, the cells were washed with PBS and $1 \mathrm{ml}$ "chase medium" (Iscove's modified minimal medium with PSF) was added both to the upper and the lower compartment and conditioning of the media was allowed for a period of $6 \mathrm{~h}$. The conditioned media from both compartments were separately harvested, stored at $-80^{\circ} \mathrm{C}$, and the cells were washed once with PBS. Subsequently, again $1 \mathrm{ml}$ of chase medium, in some cases supplemented with $2 \mu \mathrm{M}$ phorbol myristate acetate (PMA), was added to each compartment [27]. After $1 \mathrm{~h}$, the conditioned media from both compartments were harvested and stored at $-80^{\circ} \mathrm{C}$ until further analysis.

Immunoprecipitation. The conditioned media of MDCK-II-cell lines, stably transfected with vWF cDNA or variants thereof, were precleared by performing two successive incubations at room temperature with gelatin-Sepharose and, subsequently, with preformed complexes of rabbit serum immunoglobulins, coupled to protein ASepharose (Pharmacia LKB, Uppsala, Sweden). Immunoprecipitation of metabolically radiolabeled vWF was carried out with preformed complexes of a rabbit anti-human vWF immunoglobulin preparation (Dakopatts, Glostrup, Denmark), coupled to protein A - Sepharose. I mmunoprecipitated material was extensively washed with a buffer, consisting of $10 \mathrm{mM}$ Tris- $\mathrm{HCl}(\mathrm{pH} 7.8), 0.15 \mathrm{M} \mathrm{NaCl}, 5 \mathrm{mM}$ EDTA, 1\% (v/v) Nonidet P-40, $10 \mathrm{mM}$ benzamidine, and $1 \mathrm{mM}$ PMSF, followed by washing with $10 \mathrm{mM}$ Tris- $\mathrm{HCl}(\mathrm{pH}$ 7.8). Samples were analyzed by SDS-polyacrylamide gel electrophoresis (SDS-PAGE) under reducing conditions [28] and the amount of VWF was quantified with a phosphor imager (Molecular Dynamics, Sunnyvale, CA).

Determination of urokinasetype plasminogen activator (u-PA) activity. An indirect assay was employed to determine u-PA activity of $50-\mu \mathrm{l}$ aliquots of conditioned media, collected after a 6 -h chase period (see metabolic labeling procedure). To that end, these aliquots were mixed with plasminogen and the plasmin-specific substrate S2251 (KabiVitrum, Stockholm, Sweden), according to the instructions of the manufacturer of the chromogenic substrate, and the generation of p-nitro aniline was recorded spectrophotometrically at $450 \mathrm{~nm}$ during an incubation period of $10 \mathrm{~h}$ [21, 22].

VWF antigen determination. The determination of the amount of VWF antigen in conditioned media of cultured cells was performed by enzymelinked immunosorbent assay (ELISA), using rabbit antiVWF immunoglobulins as a solid phase and peroxidase-conjugated rabbit anti-vWF immunoglobulins (both Dakopatts, Glostrup, Denmark) as an indicator [9].

\section{RESULTS}

\section{Immunofluorescence Analysis of MDCK-II Cells Stably Transfected with vWF CDNA}

To investigate the polarity of VWF secretion in the absence of endogenous VWF synthesis, we employed MDCK-II cells that were stably transfected with vWF 

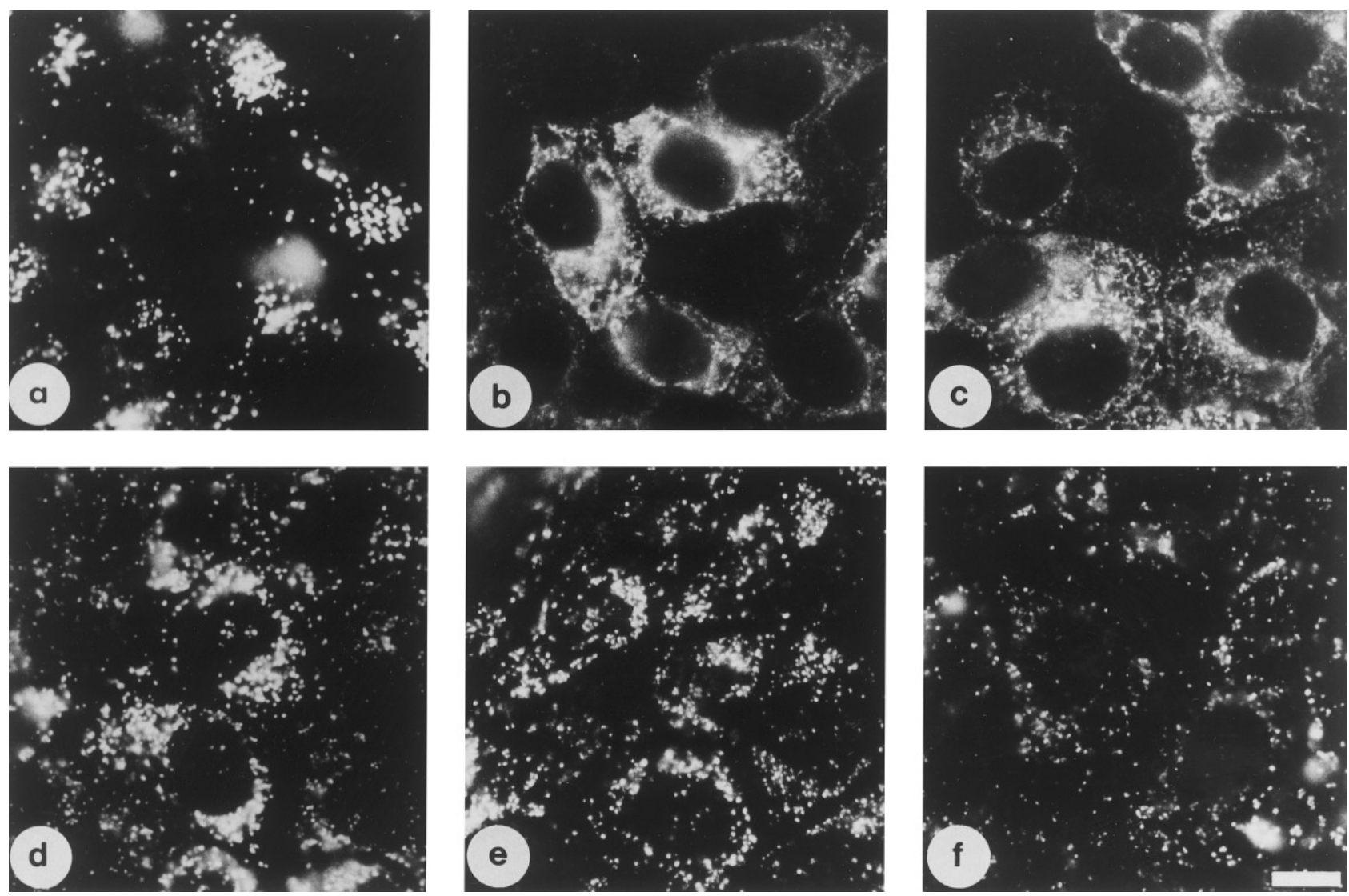

FIG. 1. I mmunofluorescence analysis of MDCK-II-vWF, MDCK-II-vWF delD1D2, and MDCK-II-vWF de1D'D3 cells. After methanol fixation, the stable transfectants were incubated both with antibodies directed against vWF and with an anti-cathepsin $D$ antiserum and subsequently with secondary antibodies as described under Materials and Methods. (a) MDCK-II-vWF, anti-vWF; (b) MDCK-IIvWF delD1D2, anti-vWF; (c) MDCK-II-vWF delD'D3, anti-vWF ; (d) MDCK-II-vWF, anti-cathepsin D; (e) MDCK-II-vWF delD1D2, anti-cathepsin D; (f) MDCK-II-vWF delD'D3, anti-cathepsin D. Bar, $5 \mu \mathrm{m}$.

cDNA. For that purpose, we developed a modification of the calcium phosphate precipitation method to integrate pcRSV VWF DNA, carrying neomycin resistance $\left(\right.$ neo $\left.^{R}\right)$, into the genome of MDCK-II cells. The resulting neo ${ }^{R}$ clones, denoted MDCK-II-VWF, were inspected by immunofluorescence by using a mixture of different murine anti-human VWF monoclonal antibodies, followed by incubation with a cy-3-labeled conjugate of goat anti-murine immunoglobulins. The analysis re vealed a reticular, perinuclear staining that is indicative for the presence of VWF in the RER and the Golgi apparatus. In addition, a punctuated pattern is observed that is reminiscent of endothelial cell-specific Weibel - Palade bodies and of the VWF-containing Weibel - Palade body-like organelles encountered in transiently transfected Monkey kidney CV-1 cells [23] (Fig. 1a). To examine the possibility that the punctuated entities of MDCK-II-vWF cells are enlarged Iysosomal structures, we labeled the cells with a rabbit polyclonal antiserum, directed against the human lysosome-specific protein cathepsin D, together with the mixture of
anti-vWF monoclonal antibodies. As secondary antibodies we used a cy3-labeled conjugate of goat antirabbit immunoglobulins and a fluorescein isothiocyanate (FITC)-labeled conjugate of goat anti-mouse immunoglobulins, respectively. Inspection of the same field clearly shows that no colocalization is observed of the anti-cathepsin D staining and the punctuate staining pattern of vWF, demonstrating that vWF is not routed to lysosomes (Fig. 1d). The lack of colocalization of the VWF-containing entities and lysosomes was confirmed by a confocal laser scanning mi croscopic (CLSM) analysis of the cy3- and FITC-labeled proteins (data not shown).

It should be noted that since MDCK-II cells form highly organized cell sheets, the immunofluorescent visualization of cathepsin D and perinuclear staining of VWF was achieved by focusing on the nuclei of the cells, whereas punctuated VWF staining, due to VWF-containing storage organelles in the cytoplasm, were conveniently visualized by focusing the microscope at a plane between the nuclei and the apical membranes. 
pro-peptide mature von Willebrand factor
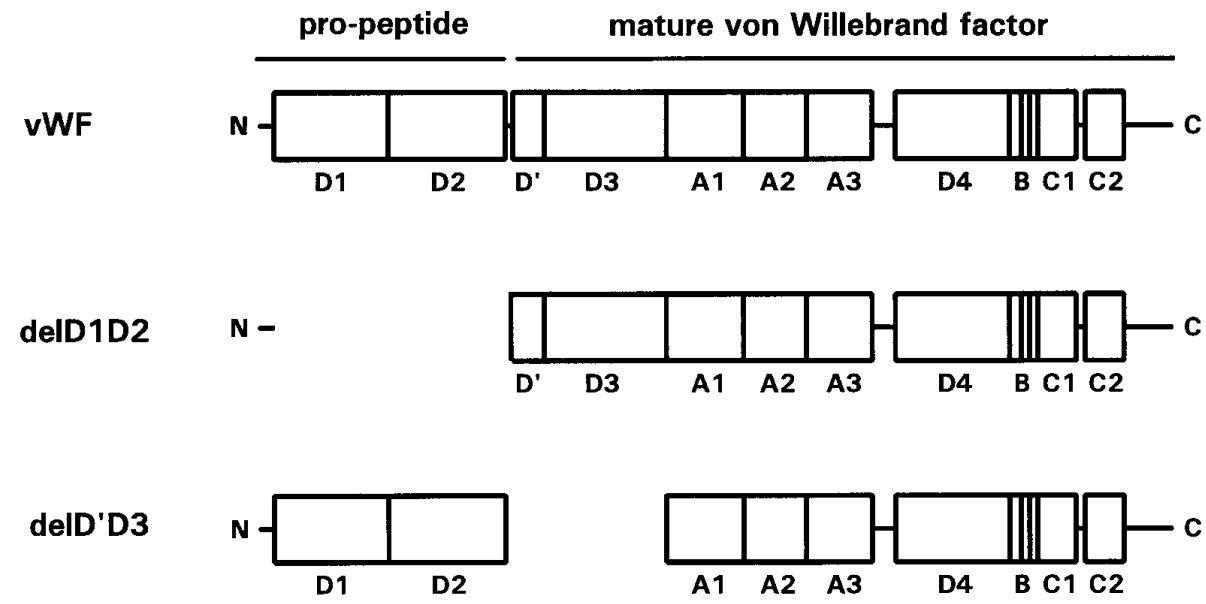

FIG. 2. Schematic representation of the domain structure of the VWF protein and of the mutant proteins VWF delD1D2 and vWF delD'D3.

\section{Analysis of MDCK-II Cells Stably Transfected with VWF deIDID2 or vWF delD'D3}

Previously we have demonstrated that storage of VWF in Weibel-Palade-like organelles can be achieved in nonendothelial cell lines, transfected with VWF CDNA. It was proposed that multimerization of VWF constitutes the triggering event for the biogenesis of these storage organelles, whereas the presence of a regulated pathway is not a requirement perse [9, 10]. The latter view is supported by the observation that vWF-transfected Monkey kidney CV-1 cells indeed generate Weibel - Palade-like storage organel les, although $\mathrm{CV}-1$ cells do not possess a regulated secretory pathway. In view of the observation that the ability of VWF to multimerize is positively correlated with the biogenesis of storage organelles, we used vWF cDNA mutants that are unable to multimerize and, hence, are not stored. This experimental design allows us to study the polarity of vWF secretion, routed exclusively by the constitutive pathway. Consequently, we constructed MDCK-II-cell lines, harboring either stable integrations of vWF delD1D2 CDNA or of vWF delD'D3 CDNA (constructs are schematically outlined in Fig. 2). The resulting cell lines are designated MDCK-II-vWF delD1D2 and MDCK-II-vWF delD'D3, respectively. The conditioned media of these cell lines were analyzed by el ectrophoresis on $2.5 \%$ (w/v) SDS agarose gels and staining with alkaline phosphataseconjugated anti-vWF antibodies (Fig. 3). The results are identical to those obtained with transiently transfected CV-1 cells [10]: only vWF dimers are formed by MDCK-II-vWF delD1D2 and MDCK-II-vWFdelD'D3 cells. In contrast, the conditioned medium of MDCKII-vWF cells displays the entire spectrum of dimers to multimers. Analysis by immunofluorescence of MDCK-II-vWF delD1D2 and MDCK-II-vWFdelD'D3 cells revealed only perinuclear and reticular staining, indicating the presence of these mutant proteins in the RER and Golgi apparatus, whereas no punctuate staining pattern is observed (Figs. $1 b$ and $1 c$ ). Finally, Iysosomal staining of MDCK-II-vWFdelD1D2 and MDCK-II-vWF del D'D3 cells with an anti-cathepsin D antiserum visualized a pattern that is indistinguishable from that of MDCK-II-VWF cells (Figs. le and 1f). Based on these morphological observations, we tentatively conclude that: (1) MDCK-II-vWF cells, that synthesize wild-type VWF, at least in part, constitutively secrete VWF, (2) in addition, a portion of the intact vWF synthesized by these cells is encountered

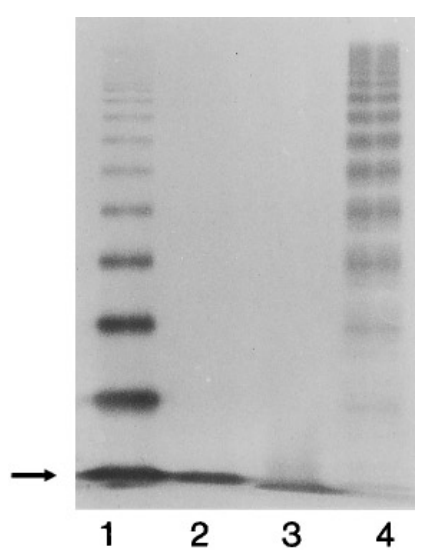

FIG. 3. Multimeric analysis of the composition of wild-type vWF and mutant proteins. Conditioned media of MDCK-II-vWF, MDCKII-vWF delD1D2, and MDCK-II-vWF delD'D3 cells were harvested and concentrated by using Centricon-30 filters. The material was analyzed by SDS - agarose gel el ectrophoresis under nonreducing conditions as described under Materials and Methods. The arrow indicates the position of vWF dimers. Bands with a lower mobility represent tetramers, hexamers, etc. It should be noted that different amounts of protein have been applied to the various lanes. Lane 1 , MDCK-II-vWF; Iane 2, MDCK-II-vWF delD1D2; Iane 3, MDCK-IIvWFdelD'D3; lane 4, plasma vWF. 

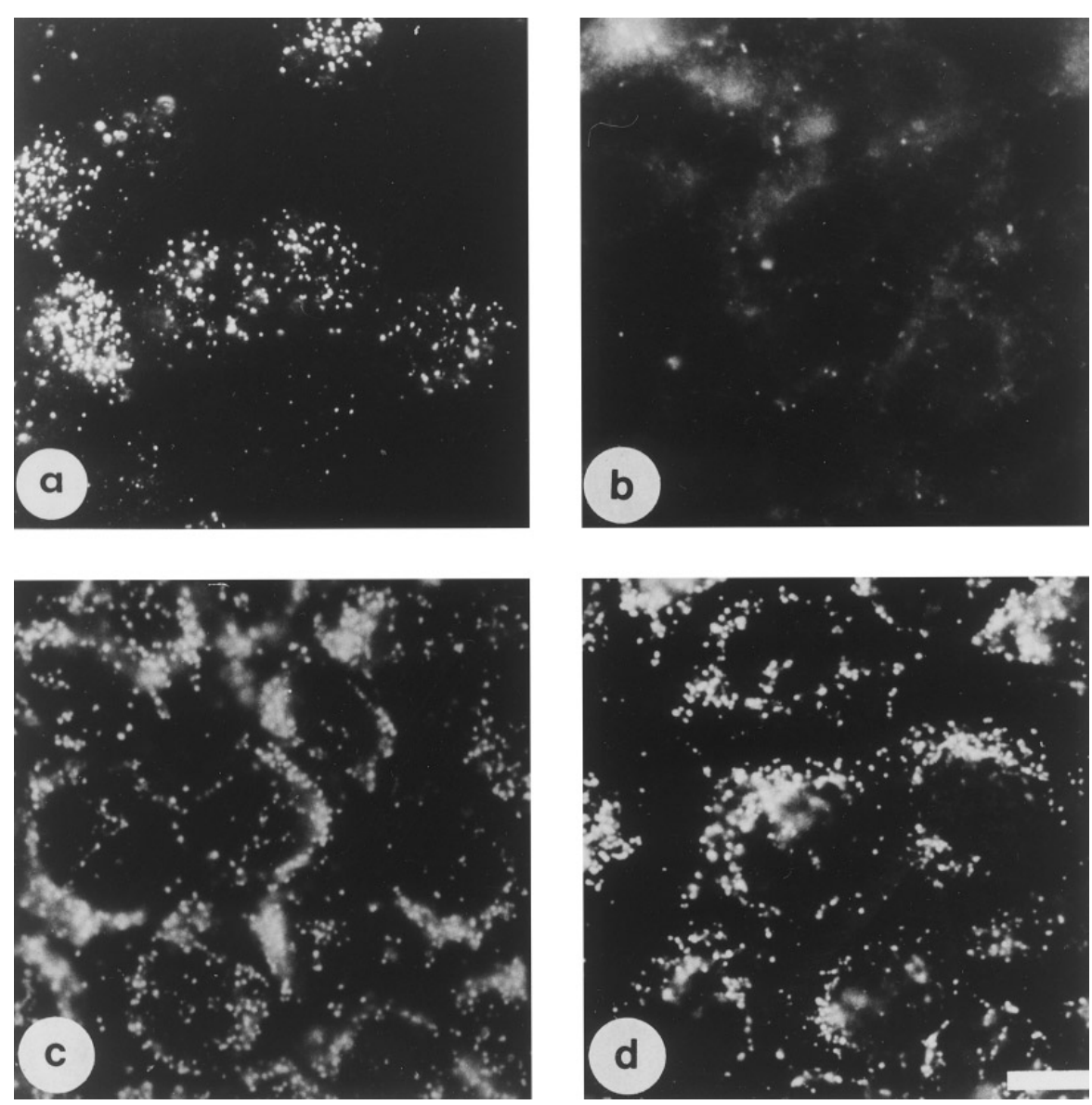

FIG. 4. Immunofluorescence analysis of MDCK-II-VWF cells before and after PMA treatment. MDCK-II-vWF cells were treated for 1 $\mathrm{h}$ with $2 \mu \mathrm{M}$ PMA and fixed with methanol. Subsequently the cells were incubated with antibodies against vWF and with an anti-cathepsin $\mathrm{D}$ antiserum as described under Materials and Methods. (a) without PMA, anti-vWF staining; (b) with $2 \mu \mathrm{M}$ of PMA, anti-vWF staining; (c) without PMA, anti-cathepsin D staining; (d) with $2 \mu \mathrm{M}$ of PMA, anti-cathepsin D staining. Bar, $5 \mu \mathrm{m}$.

in "round-shaped" entities, and (3) vWF mutants that do not multimerize are routed exclusively via the constitutive secretory pathway.

Release by Phorbol Esters of VWF Stored in MDCK-IIvWF Cells

VWF, stored in the Weibel - Palade bodies of endothelial cells, is released by activators of the protein kinase C signal transduction pathway $[6,13,14]$. To establish unambiguously that the punctuated VWF-containing entities in MDCK-II-vWF cells are Weibel-Palade body-like storage organelles, we treated the cells with phorbol myristate acetate (PMA), a potent activator of the protein kinase $C$ pathway [27]. The data of an immunofluorescence analysis are depicted in Fig. 4. In the absence of PMA, MDCK-II-vWF cells display the punctuated pattern and perinuclear staining as has been observed above (Fig. 4a). Treatment of the cells for $1 \mathrm{~h}$ with PMA results in the disappearance of the punctuated pattern (Fig. 4b). The cells that were ana- lyzed in Figs. 4a and 4b were also empl oyed to visualize the lysosomal protein cathepsin D (Figs. 4C and 4d). Clearly, treatment of MDCK-II-vWF cells with PMA did not affect the amount nor the localization of cathepsin D in these cells. This observation demonstrates that the treatment with PMA does not affect the integrity of the cells. Similarly, activation of the protein kinase $C$ pathway with phorbol dibutyrate (PDB), a soluble analogue of PMA that can be removed after the treatment of the cells, causes the disappearance of the punctuated staining pattern ( $\mathrm{Fig}$. $5 \mathrm{~b}$ ). After washing the cells to remove PDB, the punctuated pattern of vWF is restored after a recovery period of $24 \mathrm{~h}$ (Fig. 5c). These findings are interpreted as follows: (1) the punctuated vWF-containing entities in MDCK-II-vWF cells are genuine VWF-storage organelles, (2) the VWF contents of these storage organelles is released upon activation of the protein kinase $C$ signal transduction pathway, (3) upon release of VWF, MDCK-II-VWF cells are able to restore these vWF-containing storage organ- 

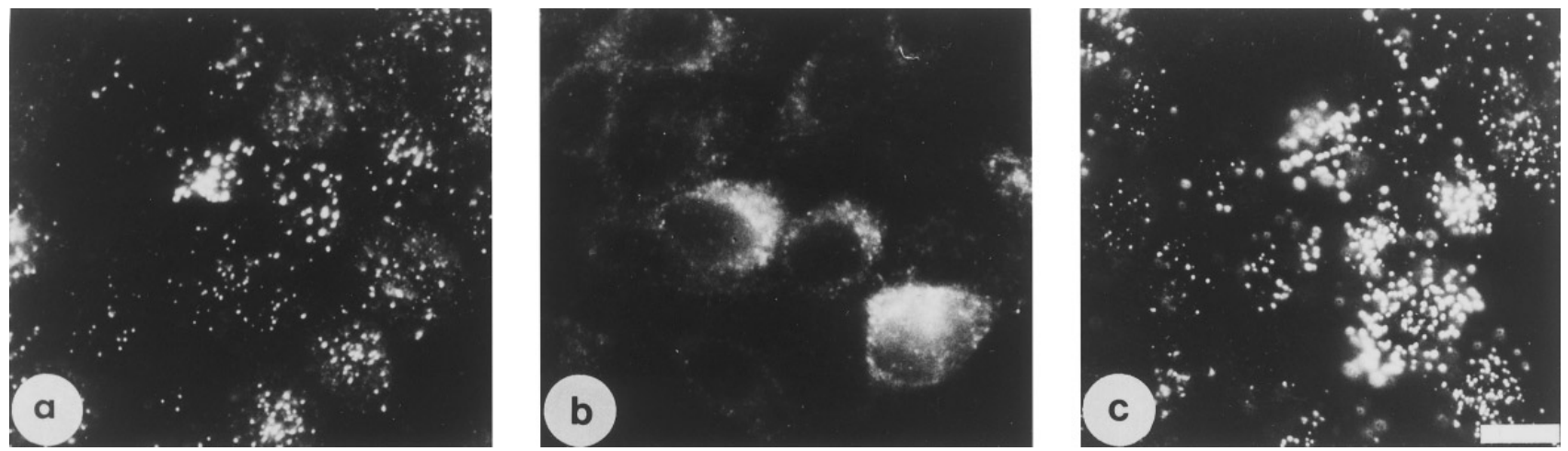

FIG. 5. I Immunofluorescent analysis of MDCK-II-vWF cells before and after PDB treatment. MDCK-II-vWF cells were treated with 2 $\mu \mathrm{M}$ PDB for $1 \mathrm{~h}$. After extensive washing the cells were cultured for another $24 \mathrm{~h}$. After methanol fixation, the cells were incubated with antibodies against VWF as described under Materials and Methods. (a) without PDB; (b) $1 \mathrm{~h}$ stimulation with $2 \mu \mathrm{M}$ PDB; (c) $24 \mathrm{~h}$ after treatment with $2 \mu \mathrm{M}$ of PDB. Bar, $5 \mu \mathrm{m}$.

elles. To our knowledge, these observations constitute the first demonstration that MDCK-II cells can store a protein that can be released by a regulated secretory pathway. It should be noted that storage of vWF had been demonstrated before in CV-1 cells that also were not known to exhibit this property [30]. However, in contrast to (stably transfected) MDCK-II-vWF cells, transiently vWF-transfected CV-1 cells did not respond to activation of the protein kinase $C$ signal transduction pathway to release VWF [10].

\section{Confocal Laser Scanning Microscopy of MDCK-II- vWF Cells}

A determination of the intracellular localization of the VWF-storage organelles in MDCK-II-vWF cells was performed by confocal laser scanning microscopy (CLSM) (Fig. 6). A gallery of photographs is shown that corresponds to a "dissection" of a number of cells in cross-sections of $1 \mu \mathrm{m}$ from the apical (upper left) to the basolateral side (lower right). This analysis reveals that all organelles are situated above the nucleus in the apical half of the cell, while staining of ER and Golgi-apparatus surrounds the nucleus. Identical results were obtained irrespective whether the cells had been grown on coverslips or on filters (data not shown). The presence of the vWF-containing storage organelles at the apical side of MDCK-II-vWF cells might be an indication that regulated vWF secretion, after activation of the protein kinase $C$ pathway, would predominantly occur to the apical compartment.

\section{Criteria for Polarity of MDCK-II-vWF Cells}

It has been well established that MDCK-II cells predominantly secrete urokinase-type plasminogen activator (u-PA) to the apical side of the cell[21, 22]. Hence, a determination of the amount of u-PA both at the apical and the basolateral side would serve as a crite- rion for maintenance of polarity and, simultaneously, for the integrity of the monolayer. Consequently, MDCK-II-vWF, MDCK-II-vWF delD1D2, and MDCKII-VWF delD'D3 cells were grown to confluency on polycarbonate filters of Transwells and the conditioned media from the apical and the basolateral compartment were collected. The data for the u-PA activity measurements of the three MDCK-II-derived cell lines are presented in Fig. 8. In agreement with the findings of others [21, 22], we observe that approximately $90 \%$ of the u-PA activity is encountered in the apical compartment

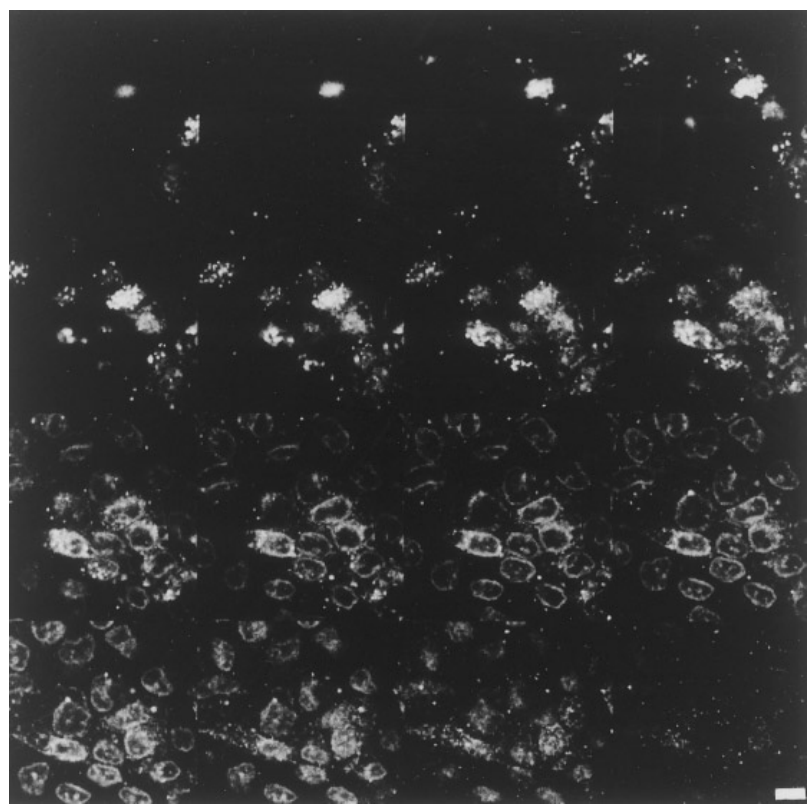

FIG. 6. Analysis of filter-grown MDCK-II-vWF cells by confocal laser scanning microscopy (CLSM). VWF was visualized with antivWF antibodies as described under Materials and Methods. Gallery of $1-\mu \mathrm{m}$ sections through the cell-layer from the apical (upper-left) to the basolateral (lower-right) side of the cells. Bar, $5 \mu \mathrm{m}$. 
and about $10 \%$ in the basolateral compartment. No significant differences were observed between the three MDCK-II-derived cell lines. A treatment of the cells for $8 \mathrm{~h}$ with PMA slightly altered the distribution of $\mathrm{u}$ PA. Under these conditions $80 \%$ of the u-PA activity is found at the apical side, whereas $20 \%$ is detected in the basolateral compartment, probably due to a minor loss of the integrity of the monolayer during this extended activation period (data not shown). However, since in our subsequent experiments an activation period of only $1 \mathrm{~h}$ has been employed, it is anticipated that the integrity of the monolayer will be fully preserved.

\section{Polarity of Regulated and Constitutive vWF Secretion by MDCK-II-vWF Cells}

We performed pulse-chase experiments to distinguish between the polarity of VWF secretion by the regulated pathway and the constitutive pathway. For that purpose, MDCK-II-vWF cells were grown for 4 days on polycarbonate filters till they reached confluency. Subsequently, the cells were radiolabeled with $\left[{ }^{35} \mathrm{~S}\right] \mathrm{methionine}$, followed by a chase period in the presence of an excess of unlabeled methionine. After the chase period, the conditioned media from the apical and the basolateral side were collected and used to analyze the constitutively secreted VWF and to measure u-PA activity. Subsequently, new chase medium was added and both compartments were incubated for $1 \mathrm{~h}$ either in the presence of $2 \mu \mathrm{M}$ PMA or in the absence of the agonist. A quantitative assessment of secreted vWF was done by immunoprecipitation of the conditioned media, followed by SDS-PAGE under reducing conditions and counting of the specific VWF protein species with a phosphor-imager. The results are presented in Figs. 7 and 8.

First, we measured u-PA activity in the media collected during the 6-h chase period. Clearly, during this experiment the integrity of the monolayer has been preserved, since $90 \%$ of the activity is present in the apical compartment, whereas $10 \%$ is found at the basolateral side (Fig. 8). Second, constitutive secretion of vWF preferentially occurs to the apical surface, since $62.0 \pm 3.8 \%$ of the VWF released during the chase period is detected in the apical compartment, whereas $38.0 \pm 3.8 \%$ is present at the basolateral side (Figs. 7a and 8). Third, regulated secretion of stored vWF after activation of the protein kinase $C$ signal transduction pathway is predominantly directed toward the apical surface, since $83.2 \pm 6.6 \%$ of the VWF is encountered at the apical side and $16.8 \pm 6.6 \%$ of the material in the basolateral compartment (Figs. 7a and 8). The amount of vWF secreted by the regulated pathway during the 1-h period is about $10-15 \%$ of that secreted by the constitutive pathway during the preceding $6 \mathrm{~h}$. Finally, it should be noted that the predominantly api-

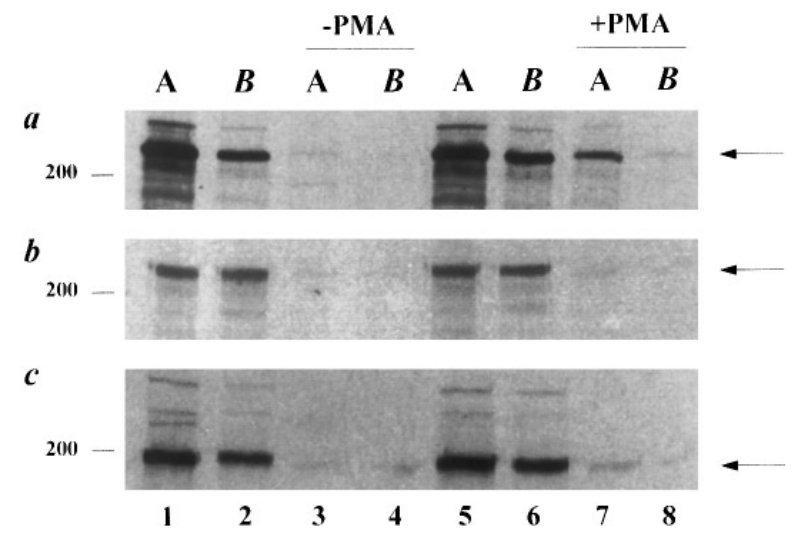

FIG. 7. Analysis of the polarity of vWF secreted either by the constitutive or by the regulated pathway. After growth of the MDCKII-vWF cell line for 4 days on polycarbonate filters in Transwells, the cells were labeled with $\left[{ }^{35}\right.$ S $]$ methionine for 3 days. Subsequently, the cells were chased with unlabeled methionine for $6 \mathrm{~h}$ and then incubated for another hour either in the presence or in the absence of $2 \mu \mathrm{M}$ PMA. VWF was immunopurified from the conditioned media and analyzed under reducing conditions by SDS - polyacrylamide gel electrophoresis. (a) MDCK-II-vWF; (b) MDCK-II-vWF delD1D2; (c) MDCK-II-vWF delD'D3. Lane 1, Constitutively secreted vWF to the apical compartment (during 6-h chase period); lane2, Constitutively secreted VWF to the basolateral compartment (during 6-h chase period); Iane 3, vWF secreted to the apical compartment in the absence of PMA (during the additional hour); lane 4, vWF secreted to the basolateral compartment in the absence of PMA (during the additional hour); lane 5, Constitutively secreted vWF to the apical compartment (during 6-h chase period); lane 6, Constitutively secreted VWF to the basolateral compartment (during 6-h chase period); Iane 7, vWF secreted to the apical compartment in the presence of PMA (during the additional hour); lane 8, VWF secreted to the basolateral compartment in the presence of PMA (during the additional hour). Symbols: A, apical; B, basolateral. Molecular weight marker is indicated at the left side.

cal secretion by the regulated pathway of MDCK-IIVWF concurs with our previous observations made by CLSM that the VWF-containing storage organelles are present between the nucleus and the apical surface (Fig. 6). We conclude that vWF routed by the regulated secretory pathway is directed toward the apical surface. In addition, constitutively secreted vWF is also preferentially released to the apical compartment.

\section{Structural Determinants of WWF Involved in Polar Secretion}

We utilized the isolated MDCK-II cell lines that harbor integrated variants of VWF CDNA, to search for structural determinants on the precursor of VWF (proVWF) that are potentially required for polar secretion. Obviously, only the polarity of secretion by the constitutive pathway can be analyzed, since the vWF variants produced by MDCK-II-vWF delD1D2 and MDCKII-vWFdelD'D3 are not stored. The pulse-chase approach outlined in the previous paragraph has been 


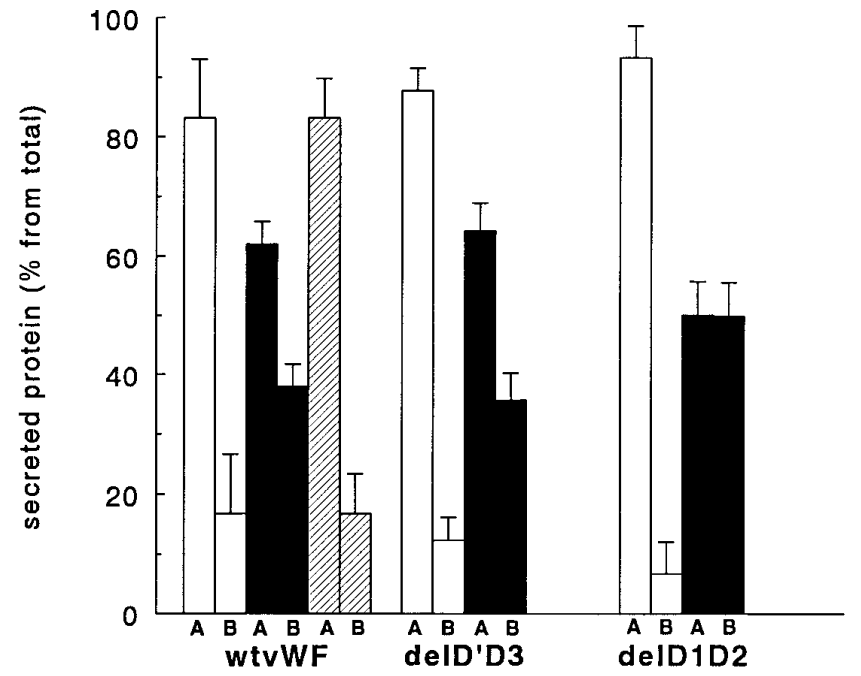

FIG. 8. Polar u-PA and vWF secretion by MDCK-II-vWF, MDCKII-vWF delD1D2, and MDCK-II-vWF delD'D3 cells. The amount of uPA secreted by cells grown on polycarbonatefilters, and subsequently secreted either to the apical (A) or the basolateral side (B) (indicated with open bars), was measured by a spectrophotometric u-PA activity assay as described under Materials and Methods. The amount of VWF constitutively secreted to both compartments (indicated with black bars) or after PMA stimulation (indicated with hatched bars) has been determined by phosphor-imager analysis of autoradiograms after SDS-polyacrylamide gel electrophoresis as shown in Fig. 7. The amount of secreted VWF (to one of the two sides) is expressed as a percentage from the total. Note that relatively little vWF is detected for the mutants VWF delD1D2 and vWF delD'D3 after treatment of the cells with PMA. The values represent three independent experiments that were performed six times. The error bars represent the standard deviations.

employed. As indicated before, the polarity of MDCKII-VWF delD1D2 and MDCK-II-vWFdelD'D3 cells is similar to that of MDCK-II-VWF, as evidenced by the apical secretion of u-PA (Fig. 8). The distribution of VWF delD'D3, secreted by the constitutive pathway, is virtually identical to that of "wild-type" vWF : $64.3 \pm$ $4.6 \%$ of the material is encountered in the apical compartment, whereas $35.7 \pm 4.6 \%$ is found at the basolateral side (Figs. 7c and 8). I nterestingly, the constitutive secretion of VWF delD1D2 does not exhibit a preference for routing to either one of the compartments. We determined that $50.1 \pm 5.7 \%$ of the VWF delD1D2 material is present at the apical side and $49.9 \pm 5.7 \%$ at the basolateral side (Figs. $7 \mathrm{~b}$ and 8). It should be noted that comparable amounts of VWF-protein are secreted by each of the three different cell-lines. VWF antigen determination by ELISA, revealed that $4.0 \mathrm{ng}$ of wildtype $v W F$ per $3 \times 10^{6}$ cells is secreted per $1 \mathrm{~h}$, whereas for vWF delD1D2 and VWF delD'D3, 6.8 and $10.1 \mathrm{ng}$ is secreted, respectively. Therefore, the observed differences in polarity are not due to different expression levels.

\section{DISCUSSION}

The adhesive property of VWF is determined by the extent of multimerization [17]. I ndirectly, the adhesive quality of vWF is dictated by the secretory pathway since release by the regulated pathway yields mostly high molecular weight multimers, whereas vWF secreted by the constitutive pathway is multimerized to a lower extent [3]. However, the cellular requirements for routing of vWF from the trans-Golgi network to storage organelles or to secretory vesicles is subject to discussion. It has been proposed that storage of vWF in Weibel-Palade body-like organelles could only be achieved in transfected cells that harbor a regulated secretion pathway, e.g., the adreno-corticotropic hormone-producing murine pituitary cell line AtT-20 or the insulin-producing cell line RIN-5 [29]. On the other hand, we have previously demonstrated that Monkey Kidney CV-1 cells, which are not known to store proteins, generate VWF-containing Weibel - Palade bodylike organelles upon transfection with vWF CDNA [10]. Similarly, in this paper we show that MDCK-II cells are also able to store vWF after transfection with vWF CDNA. To our knowledge, MDCK-II has not been identified as a cell type that harbors a regulated secretory pathway. The apparent lack of storage and subsequent regulated secretion by MDCK-II cells is supported by transient transfection studies, using cDNAs that encode a number of proteins including bovine gastric prochymosin, rat growth hormone, chicken oviduct lysozyme, and rat salivary gland $\alpha_{2}$-macroglobulin [30], that did not reveal the formation of storage organelles. In contrast, in their respective cells of origin, these hormones and other proteins are directed to storage organelles and released by the regulated secretory pathway upon treatment with appropriate agonists. However, vWF is routed to storage organelles in transfected MDCK-II cells, similar to storage in the endothelial cell-specific Weibel-Palade bodies. These results support our view that the formation of Weibel - Palade body-like storage organelles is not restricted to cells with a regulated secretory pathway, but primarily driven by determinants present on the vWF protein.

Furthermore, discrepancies have been reported on structural requirements for routing of VWF to the Weibel - Palade bodies. The data reported sofar assign either a specific sorting signal or condensation (i.e., multimerization) of the protein as the cause for the biogenesis of these organelles. In favor of the first view, it has been proposed that the propolypeptide would act as sorting signal for routing of VWF to the Weibel Palade bodies [29]. The second option is supported by the correlation between the ability to multimerize and the generation of storage organelles and, conversely, between the lack of formation of storage organelles and inability to form multimers [10]. In this report we con- 
firm the view that the mutants VWFdelD1D2 and vWF delD'D3, both mutants that lack the ability to multimerize, are also not stored in transfected MDCKII cells and are only constitutively secreted. These results are in agreement with the view that condensation (i.e., multimerization) of the protein is the triggering event for the formation of Weibel - Palade body-like organelles, similar to the condensation of hormones before storage [15]. At present, however, it cannot be excluded that the divergent concepts on the structural requirements for routing to storage organelles might be due to the use of different cell types for transfection experiments with VWF CDNA or variants thereof.

The formation of VWF-containing storage organelles in transfected MDCK-II cells, and subsequent release upon activation of the protein kinase $C$ signal transduction pathway, provides us with a model system to study the polarity of secretion by a regulated and a constitutive route. Furthermore, observations with this model system may clarify divergences that are observed on the polarity of vWF secreted by cultured human umbilical vein endothelial cells. Constitutive secretion by endothelial cells, cultured on collagen matrices, results in approximately threefold more deposition of VWF at the basolateral side than at the apical side [31]. In contrast, the constitutive secretion of VWF by endothelial cells, grown on polycarbonate filters, has been reported to be virtually nonpolar [32], whereas others found that under these conditions about $70 \%$ of the constitutively secreted VWF is encountered at the apical side [33]. Our data, using well-defined polar growing MDCK-II-vWF cells, are compatible with the latter report and demonstrate preferential apical secretion of vWF via the constitutive secretory pathway. A similar preferential sorting to the apical surface was observed for the mutant vWF delD'D3, whereas a mutant lacking the domains D1 and D2 was secreted in a nonpolar fashion. To date, a rational explanation for the observed divergences with regard to the polarity of constitutive vWF secretion, which are obtained with various systems, is not at hand. Possibly differences are due to different culturing conditions.

Finally, different results have been reported on the polarity of the release of vWF by the regulated secre tory pathway as well. Endothelial cells, grown on collagen matrices, that are treated with PMA release VWF from the Weibel - Palade bodies exclusively to the apical side [31, 34]. In contrast, endothelial cells grown on polycarbonate filters release most of the VWF to the basolateral compartment (89\%) and a minor portion (11\%) to the apical side upon PMA treatment [32]. Furthermore, the latter investigators reported on nonpolar distribution after thrombin treatment of the cells, presumably due to disruption of the integrity of the endothelial cell monolayer by the agonist. Our results with MDCK-II-vWF cells as a model system support the con- cept that release of vWF by the regulated secretory pathway is directed to the apical side of the cell, since $83.2 \pm 6.6 \%$ of the material is recovered in this compartment. This conclusion is strengthened by inspection of MDCK-II-vWF cells by means of CLSM, illustrating that stored vWF is located between the nucleus and the apical surface. Recent in vivo studies with rats, treated with the coagulation factor $\mathrm{Xa}$ and a mixture of phosphotidylcholine and phosphoditylserine to generate thrombin, support the view that vWF secreted via the regulated pathway is released at the apical side [35]. By using an electron microscopic analysis of rat aortic endothelial cells, these investigators revealed that large vWF-containing vacuoles were formed, after the treatment to generate thrombin. These vacuoles were present between the nucleus and the apical surface and are presumably formed by multiple fusions of Weibel-Palade bodies. Hence, taking the available data together, we propose that release of vWF via the regulated secretory pathway occurs predominantly at the apical side of the cell.

A comparison between the polarity of the constitutive secretion of the mutant VWF delD1D2 and that of either "wild-type" vWF or vWF delD'D3 shows that the mutant vWF delD1D2 does not display polarity of secretion. This observation may indicate that determinants, contained within the propolypeptide domain D1 and/ or D2, are involved in apical constitutive secretion. Alternatively, it cannot be excluded that removal of the propolypeptide may alter the folding of the protein and disrupt a potential routing signal. However, it should be taken into account that the biosynthesis and secretion of mutant vWF delD1D2 is similar to that of "wildtype" vWF, indicating a lack of retention of malfolded VWF delD1D2 proteins in the RER, consistent with previous observations using different expression systems $[9,10]$. Furthermore, the vWF delD1D2 proteins have fully retained the property to efficiently form dimers, suggesting that the overall folding has been maintained after mutagenesis.

To our knowledge, a specific routing consensus sequence has not been reported for polar secretion of soluble proteins. A comparison between the amino-acid sequence of the VWF domains D1 and D2 with that of uPA did not reveal obvious similarities that would suggest a routing consensus sequence (C. Hop and $\mathrm{H}$. Pannekoek, unpublished observations). Recently, it has been proposed that $\mathrm{N}$-linked glycosylation of specific amino-acid residues would be an important determinant for polar secretion of soluble proteins [36]. Taking these observations and the data reported here together, it might be speculated that either specific amino-acid residues, contained within domain D1 and/ or D2, or the $\mathrm{N}$-linked glycosylation of such residues may be involved in polar secretion of vWF. 
Dr. J an van Marle is gratefully acknowledged for his help during the CSLM experiments and Cars Gravemeijer and Cees Hersbach for their photographic assistance. This study was supported by a grant (91.126) from the Netherlands Heart Foundation.

\section{REFERENCES}

1. Ruggeri, Z. M., and Ware, J . (1992) Thromb. Haemostas. 67, 594- 599

2. Ruggeri, Z. M., and Ware, J . (1993) FASEB J . 7, 30- 316.

3. Wagner, D. D. (1990) Annu. Rev. Cell Biol. 6, 217- 246.

4. Verweij, C. L., Diergaarde, P.J ., Hart, M., and Pannekoek, H. (1986) EMBO J . 5, 1839- 1847.

5. Bonthron, D. T., Handin, R. I., Kaufman, R. J ., Wasley, L. C., Orr, E. C., Mitsock, L. M., E wenstein, B. M., Loscalzo, J ., Ginsburg, D., and Orkin, S. H. (1986) Nature 324, 270- 273.

6. Wagner, D. D., and Marder, V. J . (1984) J . Cell Biol. 99, 21232130.

7. Fretto, L.J ., Fowler, W. E., McCaslin, D. R., Erickson, H.P., and McKee, P. A. (1986) J . Biol. Chem. 261, 15679- 15689.

8. Marti, T., Rösselet, S. J., Titani, K., and Walsh, K.A. (1987) Biochemistry 26, 8099-8109.

9. Voorberg, J ., Fontijn, R., Calafat, J ., J anssen, H., van Mourik, J . A., and Pannekoek, H. (1991) J . Cell Biol. 113, 195- 205.

10. Voorberg, J ., Fontijn, R., van Mourik, J. A., and Pannekoek, H. (1990) EMBO J . 9, 797-803.

11. Weibel, E. R., and Palade, G. E. (1964) J . Cell Biol. 23, 101112.

12. Sporn, L. A., Marder, V. J ., and Wagner, D. D. (1986) Cell 46, 185- 190.

13. Loesberg, C., Gonsalves, M. D., Zandbergen, J ., Willems, C., van Aken, W. G., Stel, H. V., van Mourik, J. A., and de Groot, Ph. G. (1983) Biochim. Biophys. Acta 763, 160- 168.

14. Reinders, J. H., de Groot, Ph. G., Sixma, J . J ., and van Mourik, J . A. (1988) Haemostasis 18, 246- 261.

15. Kelly, R. B. (1985) Science 230, 25- 32.

16. Ewenstein, B. M., Warhol, M. J ., Handin, R. I., and Pober, J . S. (1987) J . Cell Biol. 104, 1423- 1433.

17. Federici, A. B., Bader, R., Coliberti, M. L., de Marco, L., and Manucci, P. M. (1989) Brit. J. Haematol. 73, 93- 99.
18. Simons, K., and Fuller, S. D. (1985) Annu. Rev. Cell Biol. 1, 243- 288.

19. Simons, K., and Wandinger-Ness, A. (1990) Cell. 62, 207-210.

20. Mostov, K.E., and Cardone, M. H. (1995) BioEssays 17, 129138.

21. Ragno, P., Estreicher, A., Gos, A., Wohlwend, A., Belin, D., and Vassali, J . D. (1992) Exp. Cell Res. 203, 236- 243.

22. Canipari, R., Zurzolo, C., Polistina, C., Garbi, C., Loj, L., Cali, G., Gentile, R., and Nitsch, L. (1992) Biochim. Biophys. Acta 1175, 1-6.

23. Voorberg, J ., Fontijn, R., Calafat, J ., J anssen, H., van Mourik, J . A., and Pannekoek, H. (1993) EMBO J . 12, 749- 758.

24. Graham, F. L., and van der Eb, A. J . (1973) Virology 52, 456467.

25. Raines, G., Aumann, H., Sykes, S., and Street, A. (1990) Thromb. Res. 60, 201-212.

26. Hansson, S. H., Simons, K., and van Meer, G. (1986) EMBO J . 5, 483- 489.

27. Holm, P. K., Eker, P., Sandvig, K., and van Deurs, B. (1995) Exp. Cell Res. 217, 157- 168.

28. Laemmli, U. K. (1970) Nature 227, 680-685.

29. Wagner, D. D., Saffaripour, S., Bonfanti, R., Sadler, J.W., Cramer, E. M., Chapman, B., and Mayadas, T. M. (1991) Cell 46, 403- 413.

30. Gottlieb, T.A., Beaudry, G., Rizzolo, L., Colman, A., Rindler, M., Adesnik, M., and Sabatini, D. D. (1986) Proc. Natl. Acad. Sci USA 83, 2100- 2104.

31. van Buul-Wortelboer, M.F., Brinkman, H-J. M., Reinders, J. H., van Aken, W. G., and van Mourik, J . A. (1989) Biochim. Biophys. Acta 1011, 129-133.

32. Sporn, L. A., Marder, V.J ., and Wagner, D. D. (1989) J . Cell Biol. 108, 1283- 1289.

33. Naharara, N., Enden, T., Wijger, M., and Prydz, H. (1994) Arterioscler. Thromb. 14, 1815- 1820.

34. Hakkert, B. C., Rentenaar, J . M., and van Mourik, J . A. (1992) Br. J . Haematol. 80, 495- 503.

35. Richardson, M., Tinlin, A., Senis, Y., and Giles, A. R. (1994) Arterioscler. Thromb. 14, 990- 999.

36. Scheiffele, P., Peränen, J ., and Simons, K. (1996) Nature 378, 96- 98 . 